\title{
Análisis de la calidad en el servicio y satisfacción de los usuarios en dos hoteles cinco estrellas de la ciudad de Cartagena (Colombia) mediante un modelo de ecuaciones estructurales
}

\author{
Analysis of quality of service and customer satisfaction in two five-star hotels \\ in the city of Cartagena (Colombia) by structural equation model
}

Juan C. Vergara $^{1} \quad$ Víctor M. Quesada $^{1} \quad$ Ingrid Blanco $^{2}$

Recibido 5 de abril de 2011, aceptado 17 de octubre de 2011

Received: April 5, 2011 Accepted: October 17, 2011

\begin{abstract}
RESUMEN
Los modelos de ecuaciones estructurales se han utilizado ampliamente para el análisis de la calidad del servicio en diversas entidades, demostrando su adaptabilidad y eficacia a la hora de determinar las variables que afectan la satisfacción del cliente. Este artículo propone el uso de un modelo de ecuaciones estructurales para determinar la calidad en el servicio ofrecido por dos hoteles de la ciudad de Cartagena de Indias clasificados en la categoría de cinco estrellas, combinando el modelo de Haemoon Oh (1999) con el instrumento original de Parasuraman, Zeithalm y Berry planteado en 1985. El resultado es un diagnóstico general de las variables que mayor influencia ejercen sobre la satisfacción de los clientes y la motivación a recomendar los servicios a otras personas.
\end{abstract}

Palabras clave: Ecuaciones estructurales, calidad del servicio, satisfacción del cliente, percepciones, hoteles.

\section{ABSTRACT}

Structural equation models have been widely used to analyze the quality of service in various organizations, demonstrating its adaptability and effectiveness in determining the variables that affect customer satisfaction. This article proposes the use of structural equation modeling to determine the quality of service offered by two hotels in the city of Cartagena de Indias classified as five stars, combining Haemoon Oh model (1999), with the original instrument of Parasuraman, Zeithalm and Berry raised in 1985. The result is a general analysis of the variables that most influence on customer satisfaction and motivation to recommend services to others.

Keywords: Structural equation, service quality, customer satisfaction, perceptions, hotels.

\section{INTRODUCCIÓN}

Las características geográficas, sus atractivos naturales y culturales, su riqueza histórica, instalaciones hoteleras, sus vías y puertos, entre otros, convierten a Cartagena de Indias D.T. y C. en uno de los destinos principales de veraneo y negocios en el mar Caribe. En el diagnóstico situacional del sector turístico realizado por la Alcaldía Mayor de
Cartagena [1] se enfatiza en la vocación turística de la ciudad como generadora de ingresos, fuentes de empleo y promotora de su desarrollo, pero a su vez plantea varios retos de sostenimiento y mejora competitiva frente a destinos similares, que cuentan con mayor inversión en infraestructura y promoción. Cartagena se proyecta como destino turístico certificado, y cuenta actualmente con varios planes de expansión en infraestructura, que permitirán

1 Universidad de Cartagena. Facultad de Ciencias Económicas. Grupo métodos cuantitativos de Gestión. Centro, Calle de la Universidad N 36-100. Sede San Agustín. Cartagena, Colombia. E-mail: jvergaras@unicartagena.edu.co; vquezadai@unicartagena.edu.co

2 Universidad de Cartagena. Facultad de Ciencias Económicas. Grupo Calidad en la Educación-Reformas. Centro, Calle de la Universidad N 36-100. Sede San Agustín. Cartagena, Colombia. E-mail: iblancoh@unicartagena.edu.co 
una oferta de acomodación y hoteles mucho más competentes [2]. La calidad en el servicio deberá convertirse en el tema de interés crucial para las agencias de viajes, gremios y autoridades que deseen consolidar el sector, y como un mecanismo de evaluación de la satisfacción de los usuarios [3]. En este sentido, la calidad en el servicio ofrecida por un hotel se ve reflejada en la conformidad y la satisfacción que experimentan los usuarios sobre los diferentes servicios prestados por éste. Este artículo conjetura sobre el grado de satisfacción que presentan los usuarios en dos hoteles de la ciudad de Cartagena de Indias (Colombia) clasificados en la categoría cinco estrellas (en la ciudad se puede contar un total de 15 hoteles con esta clasificación). Para esto se hace uso de un esquema basado en el modelo Service Quality (ServQual) abstrayendo las dimensiones propuestas por Parasuraman, Zeithalm y Berry [4], el cual ha sido utilizado por organizaciones para evaluar y mejorar su calidad en la prestación de servicios, y la hipótesis empleada por Oh [5], resuelta mediante el uso de ecuaciones estructurales.

Un estudio de esta naturaleza implica la realización de una encuesta a un grupo representativo de clientes (en este caso a usuarios de los servicios del hotel) con el fin de verificar si sus necesidades son atendidas satisfactoriamente por parte de la empresa. A partir de la escala y dimensiones basadas en el modelo ServQual, se han realizado investigaciones con el fin de medir la calidad en el servicio en hoteles [6]. En el ámbito de la aplicación de modelos de ecuaciones estructurales en hoteles, muchos autores han intentado mejorar o adaptar el modelo para evaluar las expectativas y las percepciones de los clientes que reciben los servicios.

\section{REVISIÓN DE LA LITERATURA}

Parasuraman, Zeithalm y Berry [4] introdujeron una escala de 22 ítems que denominaron ServQual, para la medición de la calidad del servicio; este modelo ha sido ampliamente adoptado en el área industrial y comercial. La idea central del ServQual se encuentra en sus cinco dimensiones de la calidad en el servicio que se evalúan mediante técnicas indirectas de comparación entre las expectativas y la percepción posterior al rendimiento; es decir, la calidad en el servicio está dada o definida por las diferencias aritméticas entre las expectativas y percepciones del cliente a través de los 22 ítems de medición. Las medidas que representan la calidad en el servicio son indirectas en el sentido de que son los investigadores y no los sujetos mismos quienes realizan la comparación entre las expectativas y las percepciones.

El modelo ServQual propone contrastar la expectativa de los clientes frente a la adquisición de un servicio y la percepción una vez éste fue recibido [4]. A este esquema se le imputan varias críticas que giran en torno a las posibles fallas en la medición de las expectativas absolutas (estas pueden no existir o pueden no estar claramente definidas) de los clientes [7-8], basado en el principio de disconformidad. Teas [9] argumentó que la escala de ServQual para expectativas induce muchas perspectivas diferentes, que los sujetos no pueden diferenciar cuando les son proporcionadas las evaluaciones. Otro aspecto importante sujeto a debate sobre el modelo radica en que las expectativas y percepciones pueden estar relacionadas con otros factores que no están considerados, como lo es el periodo de duración del servicio [10], debido a que estos podrían no mantenerse estables en razón del tiempo [11].

Esta discusión ha motivado a los investigadores a incluir nuevas características, menciones y/o variables, adecuando el modelo original a diferentes áreas específicas [12], donde predomina la aplicación de ecuaciones estructurales. Los modelos de ecuaciones estructurales permiten el análisis de las relaciones causales entre las expectativas y percepciones [13], permitiendo al investigador proponer estrategias sobre los elementos que más afectan a la satisfacción del cliente.

Existen muchas variables que influyen en las expectativas de adquirir una estancia en un hotel determinado, prevaleciendo el tamaño, la ubicación, la categoría, los servicios ofrecidos agregados, los espacios para parqueo de vehículos y el precio [14], siendo este último aspecto una variable de tipo cuantitativa que podría afectar a su vez en la percepción de los clientes [15]. Según Wyllie [16], la calidad del servicio ofrecido por el sector turístico también se explica en gran parte por el proceso de entrega, indicador usualmente utilizado para evaluar la eficacia de un servicio en particular.

El modelo ServPerf es una variación del modelo ServQual basado en el desempeño y las percepciones, 
dejando a un lado la medición de las expectativas [17]. Cronin y Taylor [17] buscaron con este modelo determinar la relación existente entre la satisfacción del cliente y la intención de recompra, identificando un efecto causal positivo significativo entre estas dos variables. Oh [5] expone una hipótesis para medir la calidad del servicio y satisfacción de los usuarios, incluyendo una nueva variable de medición denominada comunicación boca a boca (WOM), generando un modelo propio aplicado al sector hotelero, analizado mediante ecuaciones estructurales (ver Figura 1).

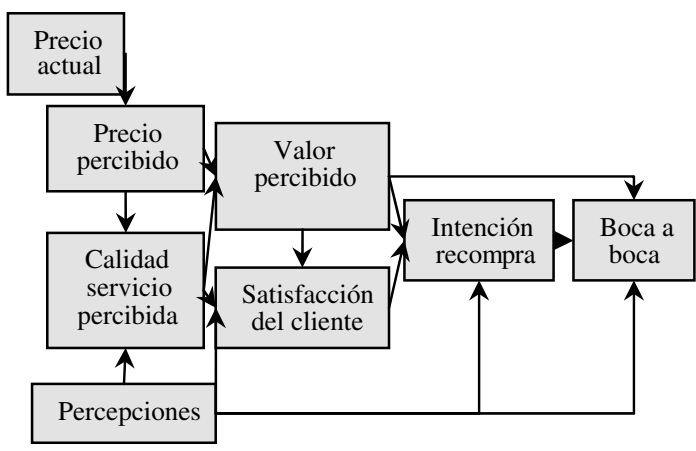

Figura 1. Modelo propuesto por Haemoon Oh [5].

En este sentido, el presente estudio evalúa la calidad del servicio ofrecido por dos hoteles categorizados en cinco estrellas, haciendo uso de ecuaciones estructurales bajo la premisa expuesta por Parasuraman, Zeithalm y Berry [4] para la definición y medición de las variables, y Oh [5] bajo su hipótesis propuesta, con la adición de nuevas variables, e incluyendo una evaluación indirecta de las expectativas mediante la valoración del precio pagado por el servicio [20].

A partir del modelo original planteado por Oh [5], se propone el siguiente esquema hipotético de relaciones entre las variables latentes consideradas en el estudio (Figura 2), permitiendo identificar el grado de correlación (con efecto positivo) entre las percepciones, la calidad percibida, la satisfacción general del cliente, la intención de recompra y la comunicación boca a boca. Se incluye además la percepción sobre el precio pagado por los servicios del hotel y su posible efecto en la calidad del servicio percibido y la satisfacción del cliente.

La propuesta final muestra un modelo simplificado con menor número de variables, excluyendo el precio

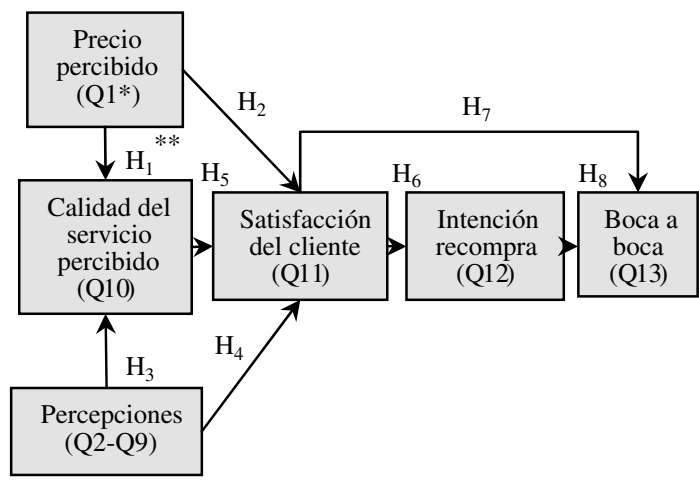

* Las variables Q1 a Q13 corresponden a las variables observadas explicadas en la Tabla 2.

** Identificación de las hipótesis planteadas en la Tabla 1.

Figura 2. Adaptación del modelo causal propuesto por Haemoon Oh [5].

actual (en acuerdo a la propuesta de los gerentes de los hoteles de no incluir preguntas relacionadas directamente con dinero) y el valor percibido. Las hipótesis planteadas en este artículo corresponden a las posibles relaciones existentes entre variables claves relacionadas con la satisfacción del cliente (ver Tabla 1).

\section{METODOLOGÍA}

\section{Muestra y datos}

Para este estudio se tomó como referencia una muestra de 350 clientes en dos hoteles cinco estrellas ubicados en la ciudad de Cartagena, con una respuesta final efectiva de 347 encuestas (equivalentes a 347 grupos de datos procesados y un error máximo de $5,26 \%$ ), procedimiento basado en un muestreo probabilístico, labor que tomó aproximadamente un mes de trabajo de campo. La encuesta aplicada contó con 21 ítems, de los cuales se desprendieron 13 preguntas clave que representan las variables observadas del modelo (los primeros ocho ítems corresponden a generalidades del encuestado).

\section{Medidas}

La percepción del precio cancelado por el usuario del hotel, relacionado con el servicio recibido, se midió considerando una escala Likert de 6 puntos, donde 1 es muy bajo y 6 muy alto. Las percepciones se evaluaron a partir de ocho preguntas (evaluadas con la misma escala Likert de 6 puntos) concernientes a: calificación de la habitación, limpieza de la habitación, limpieza de los lobbies, seguridad en las 
Tabla 1. Relación de hipótesis planteadas.

\begin{tabular}{|l|l|}
\hline Hipótesis & Relaciones \\
\hline $\mathrm{H}_{1}$ & $\begin{array}{l}\text { El precio percibido se asocia } \\
\text { positivamente con la calidad del } \\
\text { servicio percibido }\end{array}$ \\
\hline $\mathrm{H}_{2}$ & $\begin{array}{l}\text { El precio percibido se asocia } \\
\text { positivamente con la satisfacción } \\
\text { del cliente }\end{array}$ \\
\hline $\mathrm{H}_{3}$ & $\begin{array}{l}\text { Las percepciones se asocian } \\
\text { positivamente con la calidad del } \\
\text { servicio percibido }\end{array}$ \\
\hline $\mathrm{H}_{4}$ & $\begin{array}{l}\text { Las percepciones se asocian } \\
\text { positivamente con la satisfacción } \\
\text { del cliente }\end{array}$ \\
\hline $\mathrm{H}_{5}$ & $\begin{array}{l}\text { La calidad del servicio percibida } \\
\text { se asocia positivamente con la } \\
\text { satisfacción del cliente }\end{array}$ \\
\hline $\mathrm{H}_{6}$ & $\begin{array}{l}\text { La satisfacción del cliente se } \\
\text { asocia positivamente con la } \\
\text { intención de recompra }\end{array}$ \\
\hline $\mathrm{H}_{8}$ & $\begin{array}{l}\text { La satisfacción del cliente se } \\
\text { asocia positivamente con la } \\
\text { intención de recomendar los } \\
\text { servicios del hotel boca a boca }\end{array}$ \\
\hline $\begin{array}{l}\text { La intención de recompra se } \\
\text { asocia positivamente con la } \\
\text { intención de recomendar los } \\
\text { servicios del hotel boca a boca }\end{array}$ \\
\hline
\end{tabular}

instalaciones del hotel, amabilidad de los empleados, rapidez al momento del registro, capacidad de los empleados para resolver problemas y tranquilidad en la habitación.

Los encuestados calificaron la calidad en el servicio percibido usando una escala de 6 puntos, en la cual 1 representaría mucho peor de lo esperado y 6 mucho mejor de lo esperado; esta misma escala se usó para determinar el grado de satisfacción en donde 1 equivale a muy insatisfecho y 6 muy satisfecho. En cuanto a la intención de recompra, 1 se refirió a muy poco dispuesto y 6 completamente decidido; y la comunicación boca a boca se evaluó bajo el rango de 1, si se percibe como muy inseguro y 6 muy seguro. En la Tabla 2 se muestran las variables observadas propuestas consideradas en esta investigación (acorde a la numeración de la hipótesis planteada en la Figura 2; en este caso la
Tabla 2. Variables observadas referenciadas en el modelo.

\begin{tabular}{|l|}
\hline \multicolumn{1}{|c|}{ Listado de variables observadas } \\
\hline Q1. Calidad del servicio vs. valor pagado \\
\hline Q2. Calificación de la habitación \\
\hline Q3. Limpieza de la habitación \\
\hline Q4. Limpieza de los lobbies \\
\hline Q5. Seguridad en las instalaciones del hotel \\
\hline Q6. Amabilidad de los empleados \\
\hline Q7. Rapidez en el momento del registro \\
\hline $\begin{array}{l}\text { Q8. Capacidad de los empleados para resolver } \\
\text { problemas }\end{array}$ \\
\hline Q9. Tranquilidad de la habitación \\
\hline Q10. Calidad del servicio percibido \\
\hline Q11. Satisfacción del cliente \\
\hline Q12. Intención de recompra \\
\hline Q13. Comunicación boca a boca \\
\hline
\end{tabular}

variable latente percepciones es medida mediante las variables observables numeradas del 2 al 9).

Las escalas fueron validadas empleando el coeficiente Alpha de Cronbach, para medir la confiabilidad de los constructos, obteniendo un valor superior al 0,7 en cada uno de ellos. En la Tabla 3 se muestra el listado de variables latentes utilizadas en el modelo.

Tabla 3. Variables latentes referenciadas en el modelo.

\begin{tabular}{|l|}
\hline \multicolumn{1}{|c|}{ Listado de variables latentes } \\
\hline var1. Precio percibido \\
\hline var2. Percepciones \\
\hline var3. Calidad del servicio percibido \\
\hline var4. Satisfacción del cliente \\
\hline var5. Intención de recompra \\
\hline var6. Comunicación boca a boca \\
\hline
\end{tabular}

\section{Análisis}

La hipótesis propuesta en la Figura 1 fue analizada bajo un modelo de ecuaciones estructurales resuelto a través del software LISREL 8.80, aplicación con la cual se correlacionaron y describieron las variables; esta técnica de análisis permite la estimación de ecuaciones de regresión múltiple de forma simultánea, en un marco único. En particular, todas las relaciones 
Tabla 4. Resultados descriptivos de las variables observadas.

\begin{tabular}{|c|c|c|}
\hline Variable & Media & Desviación Estándar \\
\hline Q1 & 4,778 & 0,815 \\
\hline Q2 & 4,988 & 0,733 \\
\hline Q3 & 5,032 & 0,690 \\
\hline Q4 & 4,963 & 0,769 \\
\hline Q5 & 5,003 & 0,695 \\
\hline Q6 & 4,919 & 0,733 \\
\hline Q7 & 4,888 & 0,726 \\
\hline Q8 & 4,914 & 0,755 \\
\hline Q9 & 4,810 & 0,755 \\
\hline Q10 & 4,980 & 0,715 \\
\hline Q11 & 5,072 & 0,678 \\
\hline Q12 & 5,127 & 0,677 \\
\hline Q13 & 5,340 & 0,553 \\
\hline
\end{tabular}

directas e indirectas en el modelo se estiman simultáneamente y, por tanto, el método permite que todas las interrelaciones entre las variables que se evalúen estén en el mismo contexto.

\section{RESULTADOS}

Los resultados preliminares arrojaron promedios para las variables observadas comprendidos en un rango entre 4,778 y 5,340, y desviaciones estándar que no llegan a 1,000 (ver Tabla 4).

Estas variables no registraron un comportamiento normal (el valor $X^{2}$ resultado de la comparación entre la asimetría y Kurtosis por cada variable reflejó un valor muy elevado, arrojando un $\mathrm{p}<0.05$ ); se recurrió al uso del método ULS (Unweighted Least Squares), método apropiado al no requerir que los datos posean una distribución de probabilidad en particular [21].

En la Figura 3 se muestra la estructura general propuesta y los resultados estimados (factores) entre variables observadas y variables latentes arrojados por el software LISREL. Las variables identificadas como var1 y var2 (precio percibido y percepciones, respectivamente) se especificaron en el modelo como variables independientes (los comportamientos de estas tienen un efecto causal sobre las variables latentes var3, var4 y var5, consideradas como dependientes).

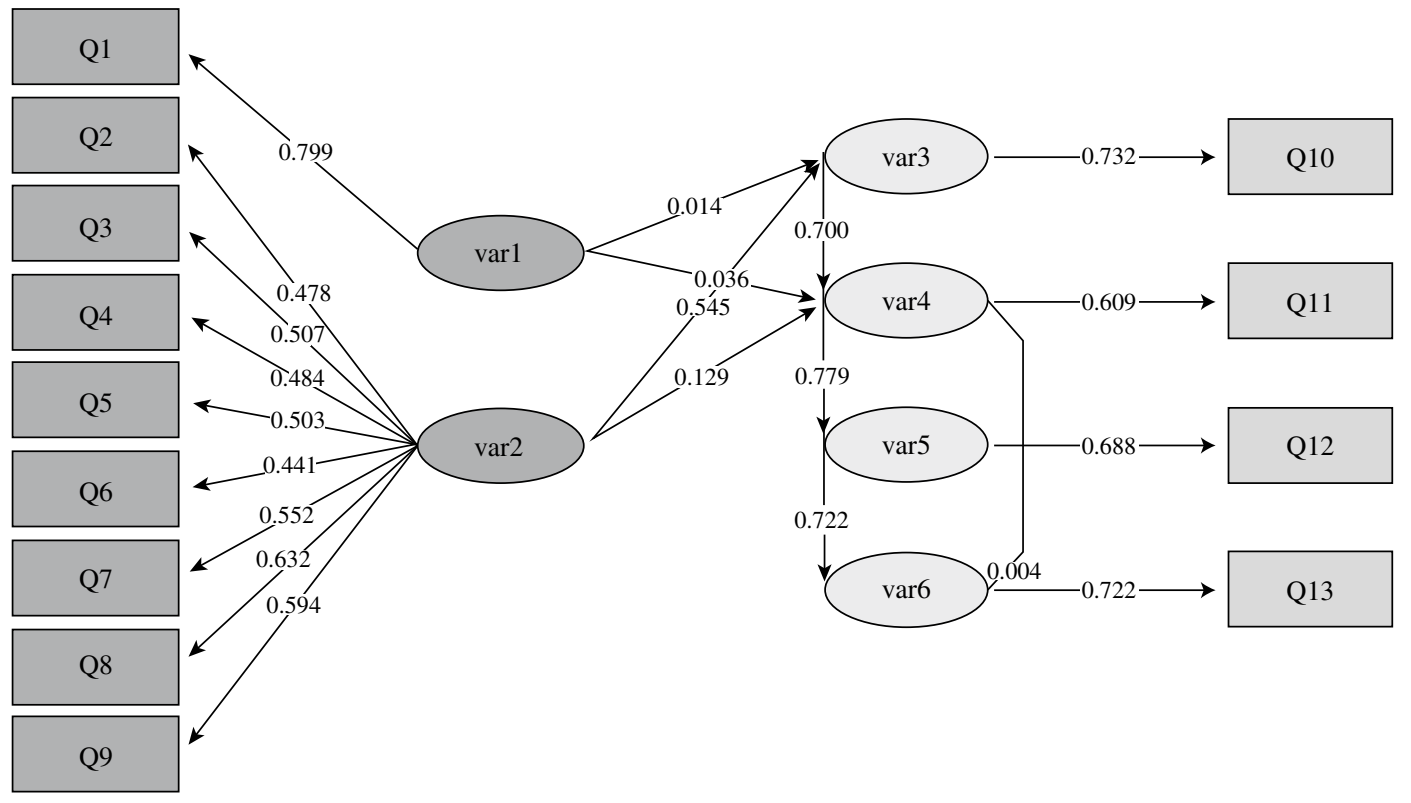

Chi-Square $=82.43, \mathrm{df}=48, \mathrm{P}-$ value $=0.00000$, RMSEA $=0.046$

* Informe gráfico mostrado por el software Lisrel 8.80. Los rectángulos identifican las variables observadas, mientras que las figuras elípticas representan las variables latentes. En color gris oscuro se muestran las variables independientes del modelo, mientras que en color gris claro se señalan las variables dependientes.

Figura 3. Variables latentes, variables observadas y sus relaciones. 
A pesar de que se obtiene un $X^{2}$ elevado (para un $\mathrm{p}<0.05$ ), se tendrán en cuenta otros indicadores para validar el modelo como la razón $X^{2} / d f$, los índices GFI (índice comparativo ajustado), NFI (índice de ajuste normado), NNFI (índice de ajuste no normado) y RMSEA (Error de Aproximación Cuadrático Medio). En el caso de la razón $X^{2} / d f$ se recomiendan valores inferiores a 4; para los índices GFI, CFI y NNFI se recomiendan cifras superiores a 0.90; y finalmente para el RMSEA por debajo de 0.05 [22, 23, 24, 25]. Los valores calculados para cada indicador confirman la validez del modelo (Tabla 5).

Tabla 5. Indicadores que confirman la validez del modelo.

\begin{tabular}{|l|l|}
\hline Indicador & Valor \\
\hline$X^{2} / d f$, & 1,720 \\
\hline GFI & 0,990 \\
\hline NFI & 0,990 \\
\hline NNFI & 1,000 \\
\hline RMSEA & 0,046 \\
\hline
\end{tabular}

Los resultados evidencian las relaciones directas e indirectas entre las variables latentes; por ejemplo, el efecto directo que tiene la calidad del servicio percibido (var3) sobre la satisfacción del cliente (var4); esta última, a su vez, afecta directamente la intención de recompra (var5) y la intención de recomendar el hotel boca a boca (var6). Por lo tanto, la calidad del servicio percibido podría afectar indirectamente la intención de recompra y la intención de recomendar al hotel (boca a boca) a través de la satisfacción del cliente.

\section{Correlaciones}

Se aplicó la prueba de bondad de ajuste sobre las correlaciones obtenidas entre las variables latentes (considerando un nivel de confianza del $95 \%$ bajo una prueba de dos colas y una correlación significativa para un valor calculado de $t>|1,96|)$. El resultado condujo a sólo tres correlaciones no significativas como se observa en la Figura 4 (entre paréntesis se muestran los valores de $t$ calculados para cada correlación).

Estableciendo las relaciones entre las variables evaluadas en este modelo, se encontró una correlación significativa entre las percepciones y la calidad del servicio percibido $(0,545)$, asumiendo un efecto proporcional entre estas variables latentes (al mejorar la evaluación de las percepciones de los clientes se tiende a aumentar la valoración en la calidad del servicio percibido). También existen correlaciones significativas entre la calidad del servicio percibido, la satisfacción del cliente, la intención de recompra y la comunicación boca a boca.

La variable precio percibido no presenta un efecto significativo sobre la variable calidad del servicio percibido, ni ésta sobre la satisfacción del cliente. Igual deducción se puede obtener en la relación causal directa entre la satisfacción del cliente y la comunicación boca a boca con una correlación cercana a cero $(0,004)$.

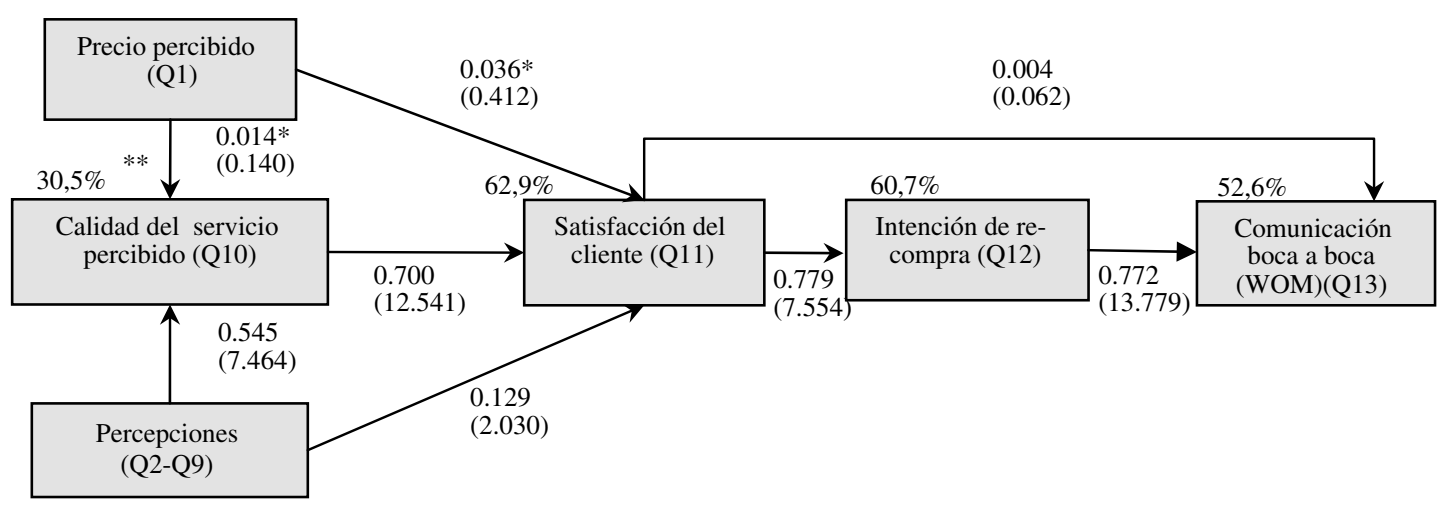

* La correlación entre las variables latentes no es significativa.

** Valor de la varianza explicada $\left(\mathrm{R}^{2}\right)$.

Figura 4. Resultados del modelo causal propuesto. 
En detalle, entre las percepciones (haciendo referencia a las variables observadas Q2 a Q9, todas ellas con correlaciones significativas) las que presentan mayor valor en su correlación se encuentran la rapidez al momento del registro, capacidad de los empleados para resolver problemas, la tranquilidad de la habitación y la limpieza de los lobbies.

El esquema propuesto explica el valor de la varianza en las variables clave del modelo: aproximadamente el 52,6\% de la varianza en la intención de recomendar el hotel boca a boca, $60,7 \%$ en la intención de recompra, $62,9 \%$ en la satisfacción del cliente y $30,5 \%$ en la calidad del servicio percibido.

\section{DISCUSIÓN}

A través de este artículo se demuestra el uso de una herramienta factible para la evaluación de la calidad en el servicio aplicado a hoteles, además se presenta una explicación razonable sobre el valor de la varianza explicada de las variables clave. Los resultados analizados refuerzan las hipótesis sobre la influencia causal entre la calidad del servicio percibida, la satisfacción del cliente, la intención de recompra y la comunicación boca a boca.

Los notables efectos del valor percibido sobre la satisfacción de los clientes son comprobados en el modelo resuelto, como se estableció en la hipótesis original, conclusión a la que han llegado autores con estudios similares en el sector como Brady y Cronin [18], Ramsaran [26], Nadiri y Hussain [27] y Salvador [28]. La variable precio percibido no tiene un efecto considerable sobre la calidad del servicio percibida y la satisfacción del cliente, alejándola de las estrategias urgentes que busquen mejorar estos dos indicadores.

La recomendación boca a boca del hotel puede ser influenciada de forma indirecta a través de la satisfacción del cliente y la calidad del servicio percibido, mismos resultados alcanzados por $\mathrm{Oh}$ [5]. En cuanto a las precepciones, alcanzan a afectar significativamente la calidad del servicio percibido y la satisfacción del cliente.

En este caso se observa que la variable precio no posee un efecto significativo sobre la calidad del servicio percibido y satisfacción del cliente, esto podría deberse a que el precio está dirigido a personas que poseen un nivel alto de ingresos, cuyo pago por el servicio no supondría una erogación fuerte en sus gastos personales.

\section{CONCLUSIONES}

En el presente estudio se utilizó una adaptación del modelo propuesto por Haemoon Oh [5] y la escala de medición propuesta en el modelo ServQual para analizar la calidad en el servicio ofrecido en dos hoteles cinco estrellas de la ciudad de Cartagena. A partir del uso del modelo, se pudo demostrar la influencia que tienen unas variables sobre otras, y el grado de incidencia de éstas a la hora de evaluar la calidad del servicio, demostrándose así la aplicabilidad del modelo en hoteles de esta categoría.

Entre las correlaciones obtenidas se pudieron destacar las percepciones con un alto índice de correlación con respecto a dos variables, en las cuales influyen significativamente. Como efecto de mayor relevancia se concluye que para poder aumentar la calidad del servicio percibido se debe hacer énfasis en las percepciones (la rapidez al momento del registro, capacidad de los empleados para resolver problemas, la tranquilidad de la habitación y la limpieza de los lobbies). También, al aumentar la calidad del servicio percibido se obtendrá un efecto positivo sobre la satisfacción de los clientes. En el estudio de referencia realizado por Oh [5] se llega a una conclusión similar.

Finalmente se demuestra que el modelo permite en forma exitosa evaluar la calidad del servicio en los hoteles, identificando los puntos clave en los cuales debe centrar la atención la firma, para mejorar la satisfacción de los clientes y aumentar la intención de recompra, y que a partir de estudios como este se podrían generar informes que faciliten la gestión de procesos, ayudando a mejorar los aspectos identificados, en los cuales se tiene alguna deficiencia, en aras de ofrecer un buen servicio con el fin de incrementar la fidelización y captación de nuevos clientes. Los esfuerzos de mejora se deben orientar hacia los aspectos claves detectados por estos tipos de estudios.

Se recomienda para futuros estudios incrementar el número de variables observadas por cada variable latente e incluir variables estructurales como la 
lealtad del cliente, la calidad general del sistema, entre otras variables técnicas. Se invita además a implementar este modelo en hoteles de diferentes categorías, contrastando los resultados.

\section{REFERENCIAS}

[1] Alcaldía Mayor de Cartagena. "Diagnóstico situacional del sector turístico. Cartagena: Plan Sectorial del Turismo de Cartagena de Indias". Alcaldía Mayor de Cartagena. Cartagena, Colombia. 2004.

[2] J. E. Arenas. "Aproximación a la Cartagena Empresarial: Un análisis de coyuntura". Eumed. $1^{\text {ra }}$ Edición. pp. 45-62. Cartagena, Colombia. ISBN: 978-84-692-7924-3. 2009.

[3] B. Prabhakaran, A. Arulraj, and V. Rajgopal, "Service Quality on Tourism: Application of Structural Equation Modeling". In Conference on Tourism in India-Challenges Ahead. Kozhikode, Kerala. 2008.

[4] A. Parasuraman, V. A. Zeithalm and L. L. Berry. "A conceptual model of service quality and its implications for future research". Journal of Marketing. Vol. 49. Issue 4, pp. 41-50. 1985. ISSN: 0022-2429. DOI: $10.2307 / 1251430$.

[5] H. Oh. "Service quality, customer satisfaction, and customer value: A holistic perspective". Hospitality Management. Vol. 18, Issue 1, pp. 67-82. 1999. ISSN: 0278-4319. DOI: 10.1016/S0278-4319(98)00047-4.

[6] S. Gadotti y A. França. "La medición de la calidad de servicio: una aplicación en empresas hoteleras". Revista Europea de Dirección y Economía de la Empresa. Vol. 18, No 2 , pp. 175-186. 2009. ISSN: 1019-6838.

[7] T. Andersson. "Another model of service quality: a model of causes and effects of service quality tested on a case within the restaurant industry". Quality Management in Service. Vol. 3, Issue 2, pp. 41-58. 1992. ISSN: 1131-6837.

[8] F. Buttle. "SERVQUAL: review, critique, research agenda". European Journal of Marketing. Vol. 30, Issue 1, pp. 8-32. 1996. ISSN : 0309-0566. DOI: 10.1108/03090569610105762.

[9] R.K. Teas. "Consumer expectations and the measurement of perceived service quality".
Journal of Professional Services Marketing. Vol. 1, Issue 1, pp. 33-53. 1993. ISSN: 0748-4623.

[10] D. Iacobucci, K. Grayson and A. Omstrom. "The calculus of service quality and customer satisfaction: theoretical and empirical differentiation and integration". Advances in Services Marketing and Management. Vol. 3, Issue 1, pp. 1-68. 1994. ISSN: 1067-5671.

[11] M.A. O'Neill. "The effects of survey timing on perceptions of service quality". Managing Service Quality. Vol. 8, Issue 2, pp. 126-132. 1998. ISSN: 0960-4529. DOI: 10.1108/09604529810206936.

[12] V. Morales, A. Hernández-Mendo y Á. Blanco. "Evaluación de la calidad en organizaciones deportivas: adaptación del modelo servqual". Revista de Psicología del Deporte. Vol. 18, Issue 2, pp. 137-150. 2009. ISSN: 1132-239X.

[13] M. Casas. "Los modelos de ecuaciones estructurales y su aplicación en el Índice Europeo de Satisfacción del Cliente". In X Jornadas Madrid 2002-ASEPUMA. pp. 1-11. Madrid, España. 2002.

[14] J. Espinet, M. Saez, G. Coenders and M. Fluvia, "Effect on prices of the attributes of holiday hotels: a hedonic prices approach". Tourism Economics. Vol. 9 Issue 2, pp. 1-14. 2003. ISSN: $1354-8166$.

[15] E. Kyoo and A. Mattila. "The Effect of Service Duration and Price (Mis)Match on Perceived Price Fairness". In 16th Graduate Students Research Conference. pp 1-8. Houston, Estados Unidos. 2011.

[16] R. Wyllie. "Tourism and society: A guide to problems and issues". Venture Publishing. $1^{\text {ra }}$ edición. New York. Estados Unidos. pp. 122127. ISBN: 978-1-892132-16-1. 2000.

[17] J. Cronin and S. Taylor. "Measuring Service Quality: A Reexamination and Extension". Journal of Marketing. Vol. 56 Issue 2, pp. 55-68. 1992. ISSN: 0022-2429. DOI: 10.2307/1252296.

[18] M. Brady and J. Cronin. "Some New Thoughts on Conceptualizing Perceived Service Quality: Hierarchical Approach". Journal of Marketing. Vol. 5 Issue 1, pp. 34-49. 2001. ISSN: 0022-2429. DOI: 10.1509/ jmkg.65.3.34.18334.

[19] Y. Armas and E. Gil. "Research Model of the Environmental Management in Hotels Firms". 
Revista PASOS. Vol. 8, No 4, pp. 479-492. 2010. ISSN: 1695-7121.

[20] J. Vergara y V. Quesada. "Análisis de la calidad en el servicio y satisfacción de los estudiantes de Ciencias Económicas de la Universidad de Cartagena mediante un modelo de ecuaciones estructurales". Revista REDIE. Vol. 13, No 1, pp. 108-122. 2011. ISSN: 1607-4041.

[21] A. Saghaei and R. Ghasemi. "Using Structural Equation Modeling in Causal Relationship Design for Balanced-Scorecards' Strategic Map". World Academy of Science, Engineering and Technology. Vol. 49, Issue 1, pp. 1032-1038. 2009. ISSN: 1307-8046.

[22] J.D. McCort and N. Malhotra. "A crosscultural comparison of behavioral intention models: Theoretical consideration and an empirical investigation". International Marketing Review. Vol. 18, Issue 3, pp. 235269. 2001. ISSN: 0265-1335.

[23] Y. Yoon, D. Gursoy and J. S. Chen. "Validating a tourism development theory with structural equation modeling". Tourism Management. Vol. 22, Issue 1, pp. 363-372. 2001. ISSN: 0261-5177. DOI: $10.1016 /$ S0261-5177(00)00062-5.
[24] J. Varela, R. Prat, C. Voces y A. Rial. “Una nueva escala para la evaluación de la calidad de los servicios de hostelería”. Psicothema. Vol. 18, Issue 1, pp. 135-142. 2006. ISSN: 0214-9915.

[25] A. Benito. "Desarrollo de un ejemplo de análisis factorial confirmatorio con LISREL, AMOS y SAS". En Seminario de Actualización en Investigación sobre Discapacidad SAID, pp. 1-42, Valladolid, España. 2008.

[26] R. Ramsaran-Fowdar. "Developing a service quality questionnarie for the hotel industry in Mauritius". Journal of Vacations Marketing. Vol. 13, Issue 1, pp. 19-27. 2007. ISSN: 1479-1870.

[27] H. Nadiri and K. Hussain. "Quality"-Is a Magic Word for Customer Satisfaction: A study to diagnose how tourist perceives the quality of hotel services". In 26th EuroCHRIE Conference, pp 1-10. Dubai, Emiratos Arabes Unidos. 2008.

[28] C. Salvador. "Structural Equation Models for Predicting Customer expectation, Satisfaction and Perceived-Quality Relationships". International Journal of Academic Research. Vol. 1, Issue 1, pp. 147-152. 2009. ISSN: 2075-412. 